\title{
Optimal Disturbance Rejection with Zero Steady-State Error for Nonlinear Vehicle Suspension Systems under Persistent Road Disturbances
}

\author{
Xiao-Fang Zhong, ${ }^{1}$ Shi-Yuan Han $\mathbb{D}^{1,2}$ Xi-Xin Yang, ${ }^{3}$ Yuan-Lin Guan, ${ }^{4}$ and Jin Zhou ${ }^{2}$ \\ ${ }^{1}$ School of Information Technology, Shandong Women's University, Jinan 250300, China \\ ${ }^{2}$ Shandong Provincial Key Laboratory of Networked Based Intelligent Computing, University of Jinan, Jinan 250022, China \\ ${ }^{3}$ College of Data Science and Software Technology, Qingdao University, Qingdao 266100, China \\ ${ }^{4}$ School of Mechanical Engineering, Qingdao University of Technology, Qingdao, China
}

Correspondence should be addressed to Shi-Yuan Han; ise_hansy@ujn.edu.cn

Received 3 March 2018; Accepted 3 May 2018; Published 31 May 2018

Academic Editor: Matteo Strozzi

Copyright (C) 2018 Xiao-Fang Zhong et al. This is an open access article distributed under the Creative Commons Attribution License, which permits unrestricted use, distribution, and reproduction in any medium, provided the original work is properly cited.

\begin{abstract}
The road disturbance rejection problem for vehicle active suspension involving the nonlinear characteristics is researched in this paper. A continuous-time state space of nonlinear vehicle active suspension is established first, in which the road disturbance is generated from the output of an introduced exosystem based on the ground displacement power spectral density. After that, based on the dynamics of road roughness and the internal model principle, a disturbance compensator with zero steady-state error is designed, which is related to the dynamic characteristics of road disturbance and independent of the control system model. By combining the vehicle active suspension system and the designed road disturbance compensator, an augmented system is obtained without explicit indication of road disturbance. Then by solving a series of decoupled nonlinear two-point-boundaryvalue problem and employing an iterative computing algorithm, an approximation optimal road disturbance rejection controller is obtained. Finally, the simulation results illustrate that the proposed approximation optimal road disturbance rejection controller can reduce the values of sprung mass acceleration, tire deflection, suspension deflection, and energy consumption and compensate the nonlinear behaviors of vehicle active suspension effectively.
\end{abstract}

\section{Introduction}

With the development of advanced actuator technologies, vehicle active suspension provides the basic support for active safety technology of ground vehicle $[1,2]$. In general, the road-induced vibration can be isolated from the vehicle body by using the springs and dampers installed in the passive vehicle suspension. However, the passive vehicle suspension cannot satisfy the soaring performance requirements, such as reducing the sprung mass acceleration to improve ride comfort, providing the small suspension deflection to keep the tire contract with ground, and reducing the displacement between the sprung component and unsprung component to obtain the small suspension deflection. Meanwhile, the above performance requirements are usually contradictory
[3-5]. As an effective component of ground vehicle for active safety technology, many research efforts have been devoted to developing the technology of vehicle active suspensions in recent years from flexible structure design and control strategies to satisfy the different performance requirements $[6$, 7]. Due to the contradiction among the above performance requirements, the developing control optimal algorithms have significantly contributed with small energy consumption as the core part of vehicle active suspension, such as linear quadratic optimal and risk-sensitive vibration control method [8], preview active vibration control method [9], and stochastic optimal active disturbance attenuation controller [10].

Vehicle active suspension is usually a typical nonlinear system. While designing the control strategies, the tire 
lift-off phenomenon, the spring nonlinearity, and the piecewise linear behavior of the damper must be taken into consideration $[11,12]$. In order to avoid the deterioration of ride comfort and even structural damage caused by nonlinear responses, various nonlinear compensation control strategies have been designed for nonlinear vehicle active suspension. An adaptive control scheme was designed for nonlinear vehicle active suspension based on driving state in [13]; an approximation optimal vibration controller for a $T-S$ fuzzy networked nonlinear vehicle suspension with random actuator time delay was proposed in [5]; a systematic and novel frequency-domain linear feedback control method was established for nonlinear vehicle active suspension by designing the nonlinear characteristic output spectrum in [14]; considering the varying sprung, unsprung masses, and the unknown actuator nonlinearity, a $T-S$ fuzzy slidingmode control scheme was designed in [15] for nonlinear active suspension vehicle systems; a novel adaptive hybrid controller was designed for a nonlinear vehicle seat suspension based on the sliding-mode controller and $H \infty$ control technique in [16]. While applying the optimal vibration control theory to the nonlinear vehicle active suspension, a Hamilton-Jacobi-Bellman (HJB) equation with no exact analytical solution will be introduced [17-19]. However, it is difficult to seek the solution under the external disturbance and nonlinear dynamics while designing the optimal road disturbance rejection controller.

From the perspective of isolating the road-induced vibration, the precise estimated information of road disturbance plays an important role in designing the feedforward component of control strategies [20-22]. In general, the road disturbance is related to the road surface roughness directly. Taken the random feature of road roughness into consideration, the power spectrum density is usually employed to describe the road disturbance. Various methods have been employed to reconstruct the road roughness, such as artificial neural network [23] and new cubic spline weight neural network [24]. Actually, if an accurate model for describing the road disturbance could be established, the good performance of disturbance rejection can be obtained $[25,26]$ by employing the disturbance compensator with zero steadystate error. Meanwhile, due to the random characteristics of road disturbances, the actuators need to pull down or push up together with the suspension motions. The actuator energy consumption must be taken into consideration while designing the road disturbance rejection controller.

Based on the above analysis, this paper studies the optimal road disturbance rejection problem for nonlinear vehicle active suspension. The contribution of this paper includes twofold. On the one hand, the road disturbance rejection problem is formulated as a nonlinear two-point-boundaryvalue problem for an augmented system, which is constructed by combining a designed road disturbance compensator and a nonlinear vehicle active suspension. On the other hand, by solving a series of decoupled two-point-boundary-value problem, an approximation optimal disturbance rejection control scheme is proposed for the augmented system, which includes feedback component and nonlinear compensation component. Finally, simulation results are given to illustrate

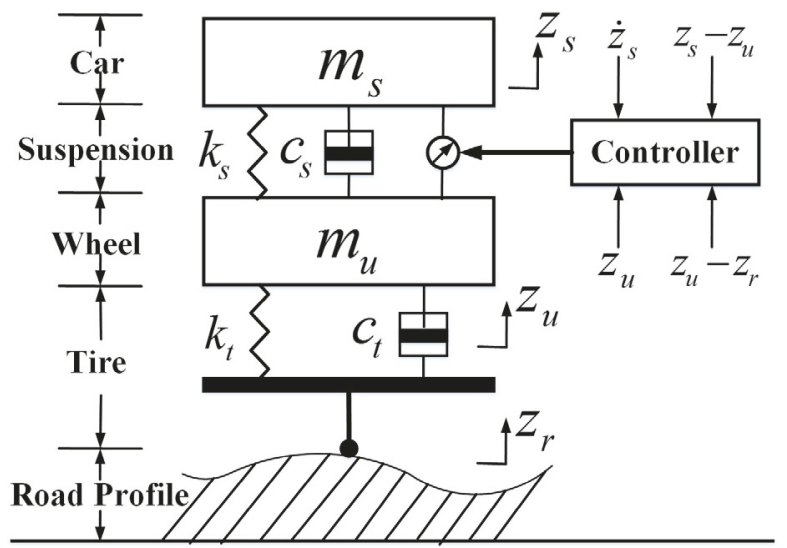

FIGURE 1: The simplified structure of quarter vehicle active suspension.

the effectiveness of improving the control performance and compensating the nonlinear behaviors of vehicle active suspension.

The rest of the article is organized as follows. The nonlinear vehicle active suspension model is established under persistent road disturbance in Section 2. In Section 3, the road disturbance rejection problem is formulated based on a designed disturbance compensator. An approximation optimal disturbance rejection controller is given in Section 4 . In Section 5, simulation results are given to illustrate the effectiveness of the proposed control law by employing a vehicle active suspension. Concluding remarks are drawn in Section 6.

\section{Modeling of Quarter Vehicle Active Suspension}

In this paper, a simplified structure of quarter vehicle active suspension with an ideal active actuator is considered, which is presented in Figure 1. The global dynamic equations of the vehicle active suspension can be described as

$$
\begin{aligned}
& c_{s}\left[\dot{z}_{s}(t)-\dot{z}_{u}(t)\right]+k_{s}\left[z_{s}(t)-z_{u}(t)\right] \\
& \quad+\varepsilon_{1} k_{s}\left[z_{s}(t)-z_{u}(t)\right]^{3}+m_{s} \ddot{z}_{s}(t)=u(t), \\
& c_{s}\left[\dot{z}_{u}(t)-\dot{z}_{s}(t)\right]+k_{s}\left[z_{u}(t)-z_{s}(t)\right] \\
& \quad+c_{t}\left[\dot{z}_{u}(t)-\dot{z}_{r}(t)\right]+k_{t}\left[z_{u}(t)-z_{r}(t)\right] \\
& \quad-\varepsilon_{2} k_{t}\left[z_{u}(t)-z_{r}(t)\right]^{3}+m_{u} \ddot{z}_{u}(t)=-u(t),
\end{aligned}
$$

where $m_{s}$ and $z_{s}(t)$ stand for the mass and displacement of sprung components; $m_{u}$ and $z_{u}(t)$ denote the mass and displacement of unsprung components, respectively; $c_{s}$ and $k_{s}$ stand for the damping and stiffness of the passive suspension components; $k_{t}$ and $c_{t}$ are the compressibility and damping of the pneumatic tire. $u(t)$ is the control force generated from an ideal hydraulic actuator; $z_{r}(t) \in R^{q}$ is the road displacement acting on the vehicle active suspension. 
Defining

$$
\begin{aligned}
& x_{1}(t)=z_{s}(t)-z_{u}(t), \\
& x_{2}(t)=z_{u}(t)-z_{r}(t), \\
& x_{3}(t)=\dot{z}_{s}(t)-z_{u}(t), \\
& x_{4}(t)=\dot{z}_{u}(t),
\end{aligned}
$$

and denoting

$$
x=\left[\begin{array}{llll}
x_{1} & x_{2} & x_{3} & x_{4}
\end{array}\right]^{T},
$$

then the continuous-time state space of nonlinear vehicle active suspension (1) can be written as

$$
\begin{aligned}
\dot{x}(t) & =\bar{A} x(t)+\bar{B} u(t)+\bar{f}(x(t))+\bar{D} v(t), \\
y_{c}(t) & =\bar{C} x(t)+E u(t), \\
y_{m}(t) & =C_{1} x(t), \\
x(0) & =x_{0},
\end{aligned}
$$

where $x(0)$ is the initial state, $v(t)=\dot{z}_{r}(t)$ denotes the road disturbance, $y_{c}(t)$ and $y_{m}(t)$ are the controlled output and the measurable output, and

$$
\begin{aligned}
& \bar{A}=\left[\begin{array}{cccc}
0 & 0 & 1 & -1 \\
0 & 0 & 0 & 1 \\
-\frac{k_{s}}{m_{s}} & 0 & -\frac{c_{s}}{m_{s}} & \frac{c_{s}}{m_{s}} \\
\frac{k_{s}}{m_{u}} & -\frac{k_{t}}{m_{u}} & \frac{c_{s}}{m_{u}} & -\frac{c_{s}+c_{t}}{m_{u}}
\end{array}\right], \\
& \bar{B}=\left[\begin{array}{c}
0 \\
0 \\
\frac{1}{m_{s}} \\
-\frac{1}{m_{u}}
\end{array}\right] \\
& C_{1}=\left[\begin{array}{llll}
1 & 0 & 0 & 0 \\
0 & 1 & 0 & 0 \\
0 & 0 & 1 & 0 \\
0 & 0 & 0 & 1
\end{array}\right], \\
& \bar{D}=\left[\begin{array}{c}
0 \\
-1 \\
0 \\
\frac{c_{t}}{m_{u}}
\end{array}\right], \\
& \bar{C}=\left[\begin{array}{cccc}
-\frac{k_{s}}{m_{s}} & 0 & -\frac{c_{s}}{m_{s}} & \frac{c_{s}}{m_{s}} \\
1 & 0 & 0 & 0 \\
0 & 1 & 0 & 0
\end{array}\right] \text {, }
\end{aligned}
$$

$$
\bar{f}=\left[\begin{array}{c}
0 \\
0 \\
\frac{-\varepsilon_{1} k_{s} \bar{x}_{1}^{3}}{m_{s}} \\
\frac{\varepsilon_{1} k_{s} \bar{x}_{1}^{3}+\varepsilon_{2} k_{t} \bar{x}_{2}^{3}}{m_{u}}
\end{array}\right],
$$$$
E=\left[\begin{array}{c}
\frac{1}{m_{s}} \\
0 \\
0
\end{array}\right]
$$

in which $\varepsilon_{1}$ is the stiffness nonlinear coefficient of spring and $\varepsilon_{2}$ denotes the spring nonlinearity, respectively.

It is assumed that the road disturbance $v(t)$ is acted on the vehicle active suspension in the vertical direction. Based on the following ground displacement power spectral density, which is described as

$$
G_{d}(\Omega)= \begin{cases}G_{d}\left(\Omega_{0}\right)\left(\frac{\Omega}{\Omega_{0}}\right)^{-n_{1}}, & \Omega \leq \Omega_{0}, \\ G_{d}\left(\Omega_{0}\right)\left(\frac{\Omega}{\Omega_{0}}\right)^{-n_{2}}, & \Omega>\Omega_{0},\end{cases}
$$

then the road disturbance $v(t)$ can be generated from the following equation:

$$
v(t)=\dot{z}_{r}(t)=\sum_{j=1}^{p} j \omega_{0} \phi_{j} \cos \left(j \omega_{0} t+\theta_{j}\right),
$$

where $\Omega$ is a spatial frequency and $\Omega_{0}=1 / 2 \pi$ is a reference frequency with road roughnesses $n_{1}$ and $n_{2}$; the stack number $p \in\left(\left(\omega_{2}-\omega_{1}\right) / \omega_{0}+1,\left(\omega_{2}-\omega_{1}\right) / \omega_{0}+2\right]$ is used to restrict the frequency range with $\left[\omega_{1}, \omega_{2}\right]=\left[\beta_{1} \omega_{n}, \beta_{2} \omega_{n}\right]$, where $\omega_{n}=$ $\sqrt{k_{s} / m_{s}}$ and $0<\beta_{1}<1<\beta_{2} ; \phi_{j}=\sqrt{2 G_{d}(j \Delta \Omega) \Delta \Omega}$ with $\Delta \Omega=2 \pi / l$, where $l$ is the length of road segment, and the initial phase $\theta_{j} \in[0,2 \pi)$ is a random phase.

By designing the road disturbance state as the vector $w(t)=\left[\xi_{1}(t), \cdots, \xi_{p}(t), \dot{\xi}_{1}(t), \cdots, \dot{\xi}_{p}(t)\right]^{\mathrm{T}}$, the road disturbance $v(t)$ can be formulated as the output of the following exosystem, which is described as

$$
\begin{aligned}
\dot{w}(t) & =G w(t), \\
v(t) & =F w(t),
\end{aligned}
$$

where

$$
\begin{aligned}
& G=\left[\begin{array}{ll}
0 & I \\
\widetilde{G} & 0
\end{array}\right], \\
& F=[\underbrace{0, \cdots, 0, \underbrace{1, \cdots, 1}_{p}}_{p}], \\
& \widetilde{G}=\operatorname{diag}\left\{-\omega_{0}^{2}, \cdots,-\left(p \omega_{0}\right)^{2}\right\},
\end{aligned}
$$


in which $\operatorname{diag}\left\{-w_{0}^{2}, \cdots,-\left(p w_{0}\right)^{2}\right\}$ represents a diagonal matrix with elements $-w_{0}^{2}, \cdots,-\left(p w_{0}\right)^{2}$ as its diagonal elements, and $I$ denotes the unit matrix.

Remark 1. While designing the optimal disturbance rejection controller, the performance requirements of vehicle active suspension must be taken into account, including the sprung mass acceleration $\ddot{z}_{s}(t)$, the tire deflection $z_{u}(t)-z_{r}(t)$, and the suspension deflection $z_{s}(t)-z_{u}(t)$. Therefore, the value of controlled output $y_{c}(t)$ must be small enough to ensure the good control performance of vehicle active suspension. Then the controlled output $y_{c}(t)$ is designed.

To obtain the main results, the following assumptions are given first.

Assumption 2. The pair $(\bar{A}, \bar{B})$ is completely controllable.

Assumption 3. Each eigenvalues $\lambda_{k}(G)$ of matrix $G$ satisfy

$$
\operatorname{rank}\left[\begin{array}{cc}
\lambda_{k}(G) I-\bar{A} & \bar{B} \\
\bar{C} & 0
\end{array}\right]=7, \quad k=1,2, \ldots, 2 p,
$$

where $I$ denotes the unit matrix.

\section{Road Disturbance Rejection \\ Problem with Zero Steady-State Error via Disturbance Compensator}

In order to compensate the road disturbance, the following disturbance compensator is designed by using the internal model principle, which is described as

$$
\begin{aligned}
& \dot{\eta}(t)=G \eta(t)-y_{m}(t), \\
& \eta(0)=\eta_{0} .
\end{aligned}
$$

Combining the nonlinear vehicle active suspension (4) with the designed disturbance compensator (11), the following augmented system can be obtained:

$$
\begin{aligned}
\dot{z}(t) & =A z(t)+f(z(t))+B u(t)+D v(t), \\
y_{c}(t) & =C z(t)+E u(t), \\
y_{m}(t) & =C_{2} z(t),
\end{aligned}
$$

where

$$
\begin{aligned}
z(t) & =\left[\begin{array}{l}
x(t) \\
\eta(t)
\end{array}\right], \\
A & =\left[\begin{array}{ll}
\bar{A} & 0 \\
-C_{1} & G
\end{array}\right], \\
f(z(t)) & =\left[\begin{array}{c}
\bar{f}(x(t)) \\
0
\end{array}\right], \\
B & =\left[\begin{array}{l}
\bar{B} \\
0
\end{array}\right],
\end{aligned}
$$

$$
\begin{aligned}
D & =\left[\begin{array}{l}
\bar{D} \\
0
\end{array}\right], \\
C & =\left[\begin{array}{ll}
\bar{C} & 0
\end{array}\right], \\
C_{2} & =\left[\begin{array}{ll}
C_{1} & 0
\end{array}\right] .
\end{aligned}
$$

By integrating the dynamic model of road disturbance into the augmented system (12), the road disturbance $v(t)$ could be set as 0 while designing the optimal controller to improve the control performance of vehicle active suspension (4). Meanwhile, as stated in Remark 1, the following infinitetime quadratic performance index is introduced, which is given by

$$
J=\frac{1}{2} \int_{0}^{\infty}\left[y_{c}^{T}(t) Q y_{c}(t)+u^{T}(t) R u(t)\right] d t
$$

where $Q$ is positive-semidefinite matrix and $R$ is positivedefinite matrix.

Then, the road disturbance rejection problem for vehicle active suspension is formulated to find an optimal road disturbance rejection controller $u^{*}(\cdot)$, which makes the quadratic performance index (14) reach the minimum value under the constrains of the augmented system (12).

Based on the necessary conditions of optimal control theory, the following nonlinear two-point-boundary-value problem for the above road disturbance rejection problem can be formulated as

$$
\begin{aligned}
\dot{z}(t)= & \left(A-B \widetilde{R}^{-1} E^{T} Q C\right) z(t)+f(z(t)) \\
& -B \widetilde{R}^{-1} B^{T} \lambda(t), \\
-\dot{\lambda}(t)= & \left(C^{T} Q C-C^{T} Q E \widetilde{R}^{-1} E^{T} Q C\right) z(t) \\
& -C^{T} Q E \widetilde{R}^{-1} B^{T} \lambda(t) \\
& +\left(A^{T}+f_{z}(t)^{T}\right) \lambda(t), \\
z(0)= & z_{0}, \\
\lambda(\infty)= & 0,
\end{aligned}
$$

where $\lambda(t)$ denotes the Lagrangian vector. Then the optimal road disturbance rejection controller for the vehicle active suspension can be designed as

$$
u^{*}(t)=-\widetilde{R}^{-1}\left(E^{T} Q C z(t)+B^{T} \lambda(t)\right)
$$

where $\widetilde{R}=R+E^{T} Q E$.

However, it is difficult to solve the analytical solution $u^{*}(t)$ (17) based on the above nonlinear two-point-boundaryvalue problem (15). In this paper, an approximation optimal road disturbance rejection controller for the vehicle active suspension is designed based on the iterative calculation approach to seek the approximation optimal solution of the nonlinear two-point-boundary-value problem (15). 


\section{Design of Approximation Optimal Road Disturbance Rejection Controller}

In order to design the approximation optimal road disturbance rejection controller, the following lemma is introduced first.

Lemma 4. The nonlinear system is described as

$$
\begin{aligned}
\dot{x}(t) & =A(t) x(t)+f(x(t)), \quad t \in t_{T}=\left(t_{0}, t_{f}\right], \\
x\left(t_{0}\right) & =x_{0},
\end{aligned}
$$

where $t_{0}$ is the initial time, $t_{f}$ denotes the terminate time, $x$ is the system state vector, and $x_{0}$ is the initial state vector. The following vector function sequences $\left\{x^{(k)}(t)\right\}$ uniformly converge to the solution of system (18), which is described as

$$
\begin{aligned}
x^{(0)}(t)= & \Phi\left(t, t_{0}\right) x_{0}, \\
x^{(k)}(t)= & \Phi\left(t, t_{0}\right) x_{0} \\
& +\int_{t_{0}}^{t}\left(\Phi(t, r) f\left(x^{(k-1)}(r)\right)\right) d r, \\
x^{(k)}\left(t_{0}\right)= & x_{0}, \quad k=1,2, \ldots, t \in t_{T}=\left(t_{0}, t_{f}\right],
\end{aligned}
$$

where $\Phi\left(t, t_{0}\right)$ is the state transfer matrix with respect to the matrix $A(t)$.

Then the approximation optimal road disturbance rejection controller is given in the following theorem.

Theorem 5. Consider the road disturbance rejection problem for a nonlinear vehicle active suspension (4) and road disturbance (8) with respect to quadratic performance index (14); the approximation optimal road disturbance rejection controller is designed as follows:

$u(t)$

$$
=-\widetilde{R}^{-1}\left(\left(E^{T} Q C+B^{T} P\right) z(t)+\lim _{k \rightarrow \infty} B^{T} g^{(k)}(t)\right),
$$

where $P$ is the unique solution of the following Riccati matrix equation:

$$
\begin{gathered}
P\left(A-B \widetilde{R}^{-1} N^{T}\right)+\left(A-B \widetilde{R}^{-1} N^{T}\right)^{T} P-P B \widetilde{R}^{-1} B^{T} P \\
-N \widetilde{R}^{-1} N^{T}+C^{T} Q C=0
\end{gathered}
$$

with $N=C^{T} Q E$. The adjoint vector sequence of $\left\{g^{(i)}(t)\right\}$ is the nonlinear compensation component, which can be obtained from

$$
\begin{aligned}
g^{(0)}(t)= & 0 \\
\dot{g}^{(k)}(t)= & \left(P B \widetilde{R}^{-1} B^{T}+N \widetilde{R}^{-1} B^{T}-A^{T}\right) g^{(k)}(t) \\
& -f_{z}^{T}\left(z^{(k-1)}(t)\right)\left(g^{(k-1)}(t)+P z^{(k-1)}(t)\right)
\end{aligned}
$$

$$
\begin{array}{r}
-\operatorname{Pf}\left(z^{(k-1)}(t)\right), \\
g^{(k)}(\infty)=0, \quad k=1,2,3, \ldots,
\end{array}
$$

where the adjoint vector sequence of $\left\{z^{(i)}(t)\right\}$ is the approximation optimal solution of the fist formula of (15), which can be obtained from

$$
\begin{aligned}
z^{(0)}(t)= & 0, \\
\dot{z}^{(k)}(t)= & \left(A-B \widetilde{R}^{-1} E^{T} Q C-B \widetilde{R}^{-1} B^{T} P\right) z^{(k)}(t) \\
& \quad+f\left(z^{(k-1)}(t)\right)-B \widetilde{R}^{-1} B^{T} g^{(k)}(t), \\
z^{(k)}(0)= & z_{0}, \quad k=1,2,3, \ldots
\end{aligned}
$$

Proof. In order to solve the nonlinear two-point-boundaryvalue problem (15), the following vector sequences are introduced, which is described as

$$
\begin{aligned}
\lambda^{(0)}(t)= & f\left(z^{(-1)}(t)\right)=0, \\
-\dot{\lambda}^{(k)}(t)= & \left(C^{T} Q C-C^{T} Q E \widetilde{R}^{-1} E^{T} Q C\right) z^{(k)}(t) \\
& +\left(A^{T}-C^{T} Q E \widetilde{R}^{-1} B^{T}\right) \lambda^{(k)}(t) \\
& +f_{z}^{T}\left(z^{(k-1)}(t)\right) \lambda^{(k-1)}(t), \\
\lambda^{(k)}(\infty)= & 0, \\
z^{(-1)}(t)= & 0, \\
\dot{z}^{(k)}(t)= & \left(A-B \widetilde{R}^{-1} E^{T} Q C\right) z^{(k)}(t)+f\left(z^{(k-1)}(t)\right) \\
& -B \widetilde{R}^{-1} B^{T} \lambda^{(k)}(t), \\
z^{(k)}(0)= & z_{0},
\end{aligned}
$$

where the adjoint vector sequence of $\left\{z^{(i)}(t)\right\}$ is introduced for obtaining the approximation solution of the second formula in (15) and the vector sequence of $\left\{\lambda^{(i)}(t)\right\}$ is designed for obtaining the approximation solution of the second formula in (15). After that, we will seek the approximation solutions of $\lambda(t)$ and $z(t)$.

It should be pointed that, at the $k$ th iteration process, the value of $z^{(k-1)}$ is with known value and viewed as the external incentive item. Then the adjoint vector sequence of $u^{(k)}(t)$ can be described as

$$
u^{(k)}(t)=-\widetilde{R}^{-1}\left(E^{T} Q C z^{(k)}(t)+B^{T} \lambda^{(k)}(t)\right) .
$$

Defining the following vector sequence for $\left\{\lambda^{(i)}(t)\right\}$ :

$$
\lambda^{(k)}(t)=P z^{(k)}(t)+g^{(k)}(t),
$$

where $P$ is the unique solution of the following Riccati matrix equation, $g^{(k)}(t)$ is the adjoint vector sequence vector of the nonlinear compensation component. Calculating the 
derivative of (26) and arranging the second formula in (24), we have

$$
\begin{aligned}
- & \lambda^{(k)}(t)=\left(C^{T} Q C-C^{T} Q E \widetilde{R}^{-1} E^{T} Q C\right) z^{(k)}(t)+\left(A^{T}\right. \\
& \left.-C^{T} Q E \widetilde{R}^{-1} B^{T}\right) \lambda^{(k)}(t)+f_{z}^{T}\left(z^{(k-1)}(t)\right) \lambda^{(k-1)}(t) \\
& =\left(C^{T} Q C-C^{T} Q E \widetilde{R}^{-1} E^{T} Q C\right. \\
& \left.+\left(A^{T} C^{T} Q E \widetilde{R}^{-1} B^{T}\right)\right) z^{(k)}(t)+\left(A^{T}\right. \\
& \left.-C^{T} Q E \widetilde{R}^{-1} B^{T}\right) g^{(k)}(t)+f_{z}^{T}\left(z^{(k-1)}(t)\right) \lambda^{(k-1)}(t) .
\end{aligned}
$$

Meanwhile, by combining the fifth formula in (24), we have

$$
\begin{aligned}
\dot{\lambda}^{(k)}(t)= & P \dot{z}^{(k)}(t)+\dot{g}^{(k)}(t) \\
= & P\left(A-B \widetilde{R}^{-1} E^{T} Q C\right) z^{(k)}(t) \\
& +P f\left(z^{(k-1)}(t)\right) \\
& -P B \widetilde{R}^{-1} B^{T}\left(P z^{(k)}(t)+g^{(k)}(t)\right)+\dot{g}^{(k)}(t) .
\end{aligned}
$$

By comparing the parameters of (27) and (28), the Riccati equation (21) and the adjoint vector sequence of $\left\{g^{(k)}(t)\right\}$ in (22) can be obtained.

Noting the values of $P, z^{(k)}(t), f\left(z^{(k)}(t)\right)$, and $f_{z}\left(z^{(k)}(t)\right)$, it can be found that (22) is a nonhomogeneous linear differential equation. Then the adjoint vector sequence of $\left\{g^{(k)}(t)\right\}$ in (22) is the solution of the following equations:

$$
\begin{aligned}
& g^{(0)}(t)=0, \\
& g^{(k)}(t)=\int_{0}^{\infty} \Phi(t, r)\left\{f_{z}^{T}\left(z^{(k-1)}(r)\right)\right. \\
& \left.\quad \times\left(g^{(k-1)}(r)+P z^{(k-1)}(r)\right)+P f\left(z^{(k-1)}(r)\right)\right\} d r, \\
& g^{(k)}(\infty)=0, \quad k=1,2,3, \ldots,
\end{aligned}
$$

where $r$ denotes the calculus variable and $\Phi$ is the transfer matrix of $\left(P B \widetilde{R}^{-1} B^{T}+N \widetilde{R}^{-1} B^{T}-A^{T}\right)$.

Therefore, in the $k$ th iteration process, the optimal road disturbance rejection controller can be described as

$$
\begin{aligned}
u^{(k)} & (t) \\
& =-\widetilde{R}^{-1}\left(\left(E^{T} Q C+B^{T} P\right) z^{(k)}(t)+B^{T} g^{(k)}(t)\right) .
\end{aligned}
$$

Then, (23) can be obtained. The solution of (23) can be described as

$$
\begin{aligned}
& g^{(0)}(t)=0, \\
& g^{(k)}(t) \\
& \quad=\int_{0}^{\infty} \psi(t, r)\left\{f_{z}^{T}\left(z^{(k-1)}(r)\right)-B \widetilde{R}^{-1} B^{T} g^{(k)}(r)\right\} d r, \\
& g^{(k)}(\infty)=0, \quad k=1,2,3, \ldots,
\end{aligned}
$$

where $\psi$ denotes the transfer matrix of $\left(A-B \widetilde{R}^{-1} E^{T} Q C-\right.$ $B \widetilde{R}^{-1} B^{T} P$ ).

Based on Lemma 4, the approximation optimal road disturbance rejection controller (20) can be obtained. The proof is completed.

Due to the infinite item $\lim _{k \rightarrow \infty} g^{(k)}(t)$, the approximation optimal road disturbance rejection controller (20) is unfeasibility. Then the feasible controller at the $M$ th iteration is chosen as the final controller, which is described as

$$
\begin{aligned}
& u^{(M)}(t) \\
& \quad=-\widetilde{R}^{-1}\left(\left(E^{T} Q C+B^{T} P\right) z^{(M)}(t)+B^{T} g^{(M)}(t)\right) .
\end{aligned}
$$

Based on the difference of the performance index value in (14) between two conjoint iteration processes, the iteration number $M$ can be decided from the following algorithm.

Algorithm 6.

Step 1. Initialization: calculate the matrix $P$ by solving the Riccati equation (21), define the iteration number as $i=1$, set the sequences vectors of $g^{(0)}(t)=z_{0}(t)=0$, and define a small positive threshold constant $\varepsilon$.

Step 2. Calculation of the nonlinear compensation component at $i$ th iteration process: make $M=i$, and calculate the value of adjoint vector $g^{(M)}(k)$ from (22).

Step 3. Calculation of the controller value at the $i$ th iteration process: calculate the adjoint sequence vector $z^{(M)}(k)$ from (23) and then obtain the value of $u^{(M)}(t)$ from (30).

Step 4. Calculation of the performance index value in the $i$ th iteration process: obtain the performance index value $J_{M}$ of (14) in the Mth iteration process, which is described as

$$
\begin{aligned}
& J^{(M)}=\frac{1}{2} \int_{0}^{\infty}\left\{\left(z^{(M)}(t)\right)^{T} C^{T} Q C z^{(M)}(t)\right. \\
& +2\left(z^{(M)}(t)\right)^{T} C^{T} Q E u^{(M)}(t) \\
& \left.+\left(u^{(M)}(t)\right)^{T} \widetilde{R} u^{(M)}(t)\right\},
\end{aligned}
$$

Step 5. Judge whether the algorithm is over: if $\left|\left(J_{M}-J_{M-1}\right)\right|$ $J_{M} \mid<\varepsilon$, then stop and output the controller $u^{(M)}(k)$; else go to Step 6.

Step 6. Let $i=i+1$, and return to Step 2 .

\section{Simulation Results}

By employing the approximation optimal road disturbance rejection controller (32) to a vehicle nonlinear active suspension, the control performance and energy consumption are discussed in this section. The parameters of nonlinear vehicle active suspension are listed in Table 1 [27]. 
TABLE 1: Parameters of active vehicle suspension.

\begin{tabular}{lccc}
\hline Parameters & Variable Symbol & Value & Unit \\
\hline Mass of Sprung & $m_{s}$ & 972.2 & $\mathrm{~kg}$ \\
Mass of Unsprung & $m_{u}$ & 113.6 & $\mathrm{~kg}$ \\
Damping of Passive Suspension & $c_{s}$ & 1095 & $\mathrm{Ns} / \mathrm{m}$ \\
Stiffness of Passive Suspension & $k_{s}$ & 42719.6 & $\mathrm{~N} / \mathrm{m}$ \\
Compressibility of Pneumatic Tire & $k_{t}$ & 101115 & $\mathrm{~N} / \mathrm{m}$ \\
Damping of Pneumatic Tire & $c_{t}$ & 14.6 & $\mathrm{Ns} / \mathrm{m}$ \\
Stiffness coefficient of Spring & $\varepsilon_{1}$ & 1 & - \\
Spring Nonlinearity & $\varepsilon_{2}$ & 1 & - \\
\hline
\end{tabular}

TABLE 2: Parameters of random road roughness.

\begin{tabular}{lccccccc}
\hline Variable & $n_{1}$ & $n_{2}$ & $\beta_{1}$ & $\beta_{2}$ & $w_{n}$ & $v_{0}$ & $l$ \\
\hline Value & 2 & 1.4 & 0.45 & 5 & 2.12 & $20 \mathrm{~m} / \mathrm{s}$ & $400 \mathrm{~m}$ \\
\hline
\end{tabular}

Then the matrices of nonlinear vehicle active suspension in (5) are displayed as follows:

$$
\begin{aligned}
& \bar{A}=\left[\begin{array}{cccc}
0.0000 & 0.0000 & 1.0000 & -1.0000 \\
0.0000 & 0.0000 & 0.0000 & 1.0000 \\
-4.4838 & 0.0000 & -0.1149 & 0.1149 \\
38.3727 & -90.8362 & 0.9836 & -0.9967
\end{array}\right], \\
& \bar{C}=\left[\begin{array}{cccc}
-4.4838 & 0.0000 & -0.1149 & 0.1149 \\
1.0000 & 0.0000 & 0.0000 & 0.0000 \\
0.0000 & 1.0000 & 0.0000 & 0.0000
\end{array}\right], \\
& \bar{B}=10^{-3} \times\left[\begin{array}{l}
0.0000 \\
0.1050 \\
-0.8982
\end{array}\right], \\
& \bar{D}=\left[\begin{array}{c}
0.0000 \\
-1.0000 \\
0.0000 \\
0.0131
\end{array}\right], \\
& E=10^{-3} \times\left[\begin{array}{l}
0.1050 \\
0.0000 \\
0.0000
\end{array}\right] .
\end{aligned}
$$

Meanwhile, the parameters of road disturbance $v(t)$ in (7) are given in Table 2 .

According to the road displacement $z_{r}(t)$ in (7) and the road disturbance $v(t)$ in (8), the curves of the road displacement and the road disturbance are displayed in Figures 2 and 3, respectively. The control performance index (14) is selected as $Q=10^{7} \times \operatorname{diag}\{1,2,4\}, R=215.07$.

Applying the proposed approximation optimal road disturbance rejection controller (32) and the designed Algorithm 6 to the above nonlinear vehicle active suspension,

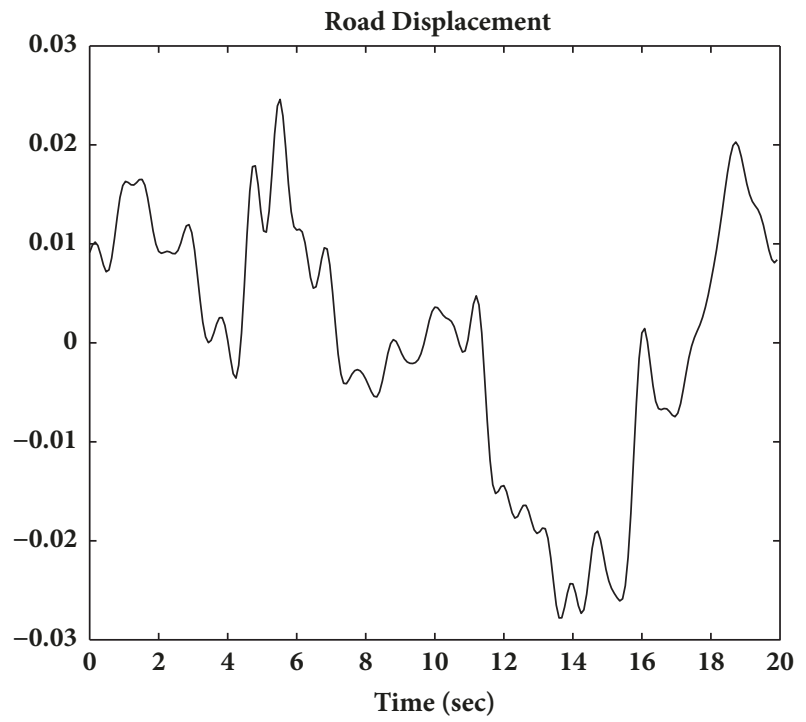

Figure 2: The curve of the road displacement $z_{r}(t)$.

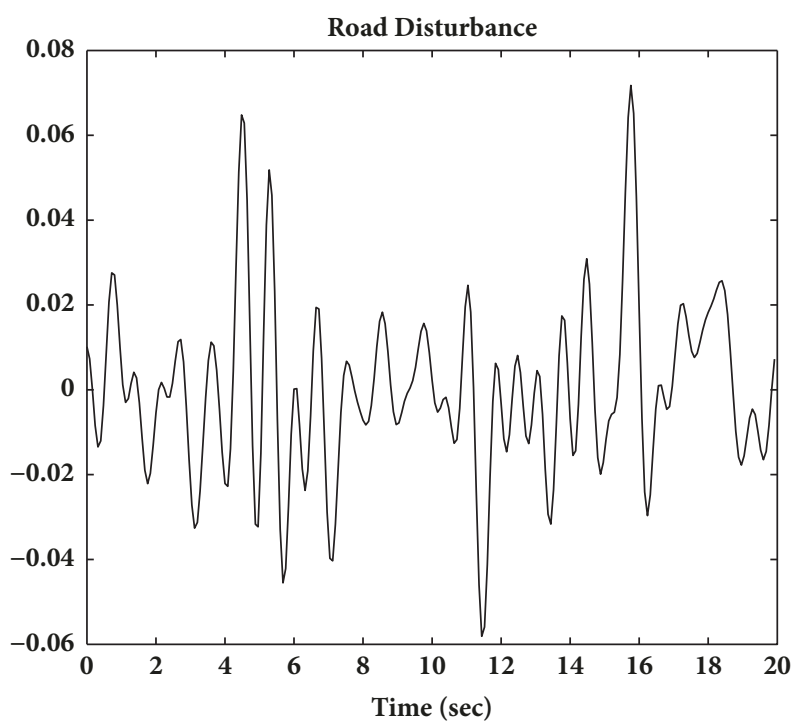

Figure 3: The curve of the road disturbance $v(t)$. 


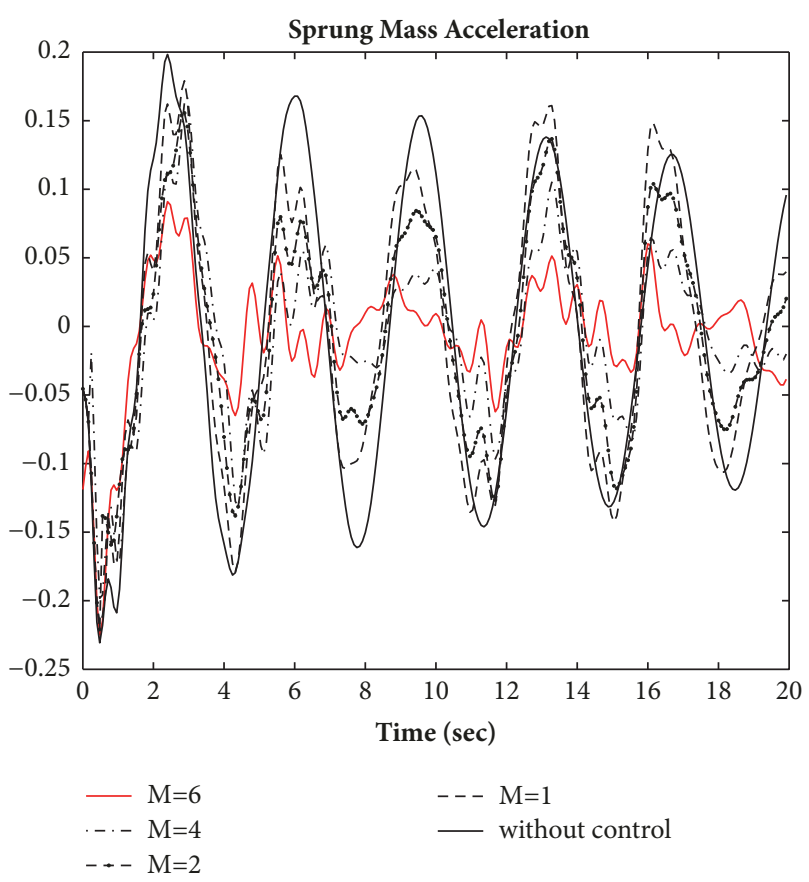

FIGURE 4: The curve of the sprung mass acceleration $\ddot{z}_{s}(t)$.

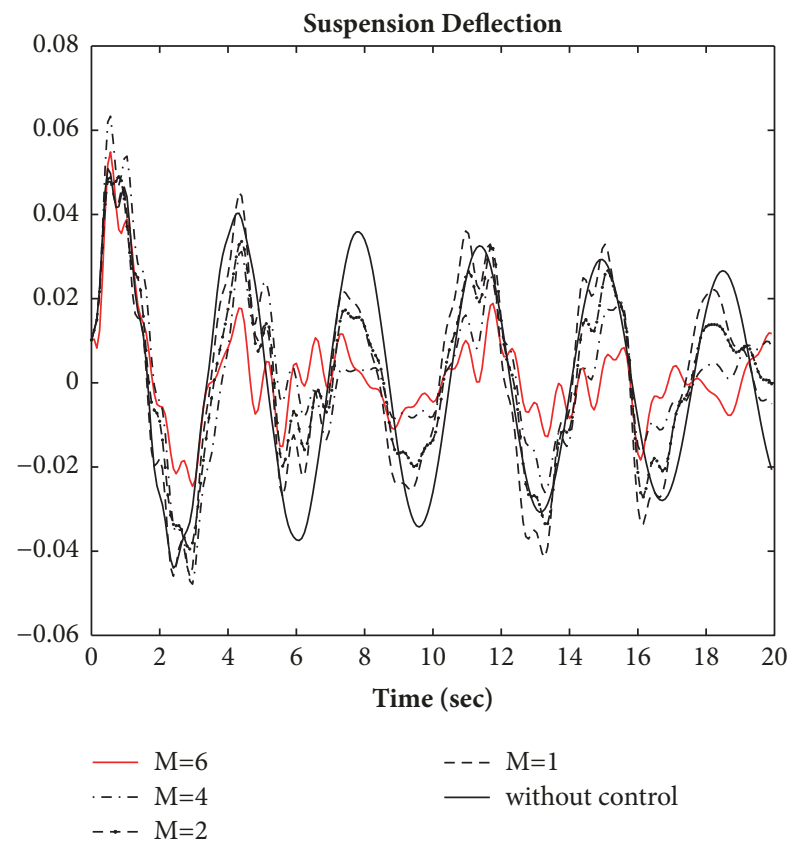

FIgURE 5: The curve of the suspension deflection $z_{s}(t)-z_{u}(t)$.

the termination condition is satisfied in the iteration process at $M=6$. In order to show the effectiveness of the proposed approximation optimal road disturbance rejection controller more clearly, the curves of the sprung mass acceleration $\ddot{z}_{s}(t)$, the suspension deflection $z_{s}(t)-z_{u}(t)$, the tire deflection $z_{u}(t)-z_{r}(t)$, and the control force are displayed in Figures 3-6, in which the simulation results in the iteration processes at $M=1,2,4$, and 6 under

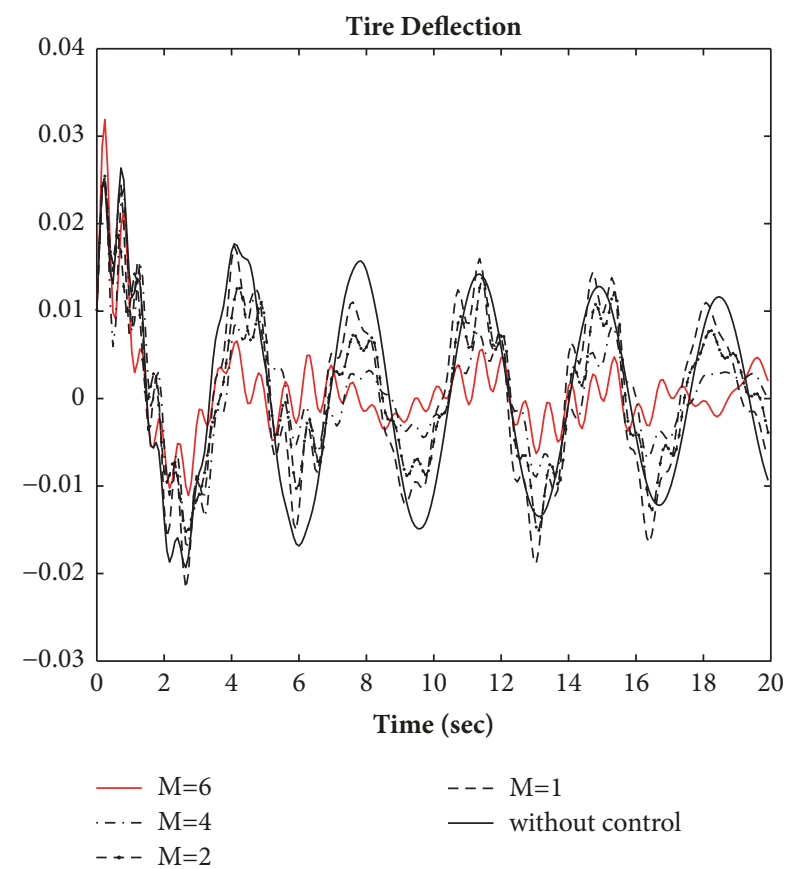

Figure 6: The curve of the tire deflection $z_{u}(t)-z_{r}(t)$.

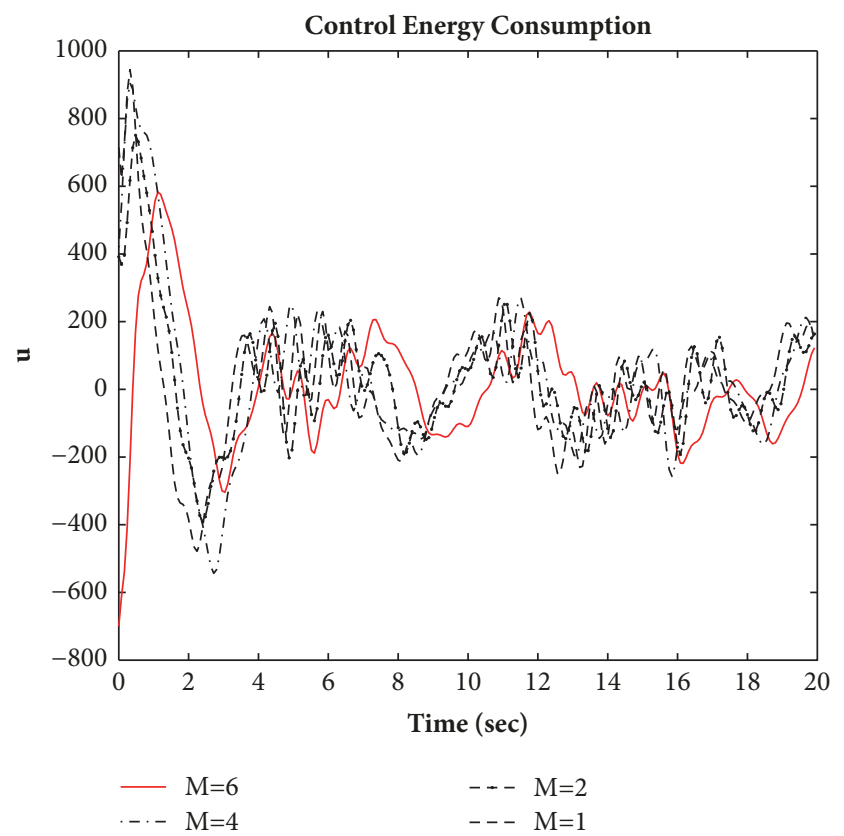

Figure 7: The curve of the control energy consumption $u(t)$.

proposed control scheme are compared with the open-loop one.

By analyzing Figures $4-7$, the control performance is improved effectively under the approximation optimal disturbance rejection controller (32). Meanwhile, the energy consumption of the approximation optimal road disturbance rejection controller (32) is with small value. Therefore, the approximation optimal road disturbance rejection controller 
can compensate the nonlinear dynamic of vehicle active suspension, satisfy the control requirements, and eliminate the road disturbances effectively with small energy consumption.

\section{Conclusions}

An approximation optimal disturbance road rejection controller was proposed for a nonlinear vehicle active suspension under persistent road disturbance, which constitutes of the feedback terms and the compensation terms for nonlinear behaviors. First, a disturbance compensator with zero steadystate error was introduced based on the ground displacement power spectral density. After that, an augmented system was designed without explicit indication of road disturbance by combining the vehicle active suspension and the designed disturbance compensator. By solving a decoupled nonlinear two-point-boundary-value problem, an approximation optimal road disturbance rejection controller was obtained from a Riccati equation and a vector sequence of nonlinear compensation terms. Applying the proposed approximation optimal road disturbance rejection controller to a nonlinear vehicle active suspension, the performance requirements were satisfied significantly with small energy consumption.

The main contribution of this paper is under the assumption that the actuator of vehicle active suspension is idealized and the dynamic road disturbance can be obtained from a road roughness with known values. One aspect of our future work will focus on the vibration controller for vehicle active suspension considering the dynamic behaviors of actuator. On the other hand, the intelligent sensors for road disturbance will be designed based on the road recognition methods by using intelligent pattern recognition theory.

\section{Data Availability}

The simulation results are from MATLAB. Readers can request the results of this article by emailing the corresponding author.

\section{Conflicts of Interest}

The authors declare that they have no conflicts of interest.

\section{Acknowledgments}

This work is supported by the Natural Science Foundation of Shandong Province (ZR2017MF044), the Shandong Province Key Research and Development Program (2018GGX101016, 2018GGX101048, and 2017GGX10144), the Shandong Province Higher Educational Science and Technology Program (J17KA047, J16LN07, J16LB06, and J15LN13), and the Natural Science Foundation of China (61671220 and 61702217).

\section{References}

[1] H. E. Tseng and D. Hrovat, "State of the art survey: active and semi-active suspension control," Vehicle System Dynamics, vol. 53, no. 7, pp. 1034-1062, 2015.
[2] J. Cao, H. Liu, P. Li, and D. J. Brown, "State of the art in vehicle active suspension adaptive control systems based on intelligent methodologies," IEEE Transactions on Intelligent Transportation Systems, vol. 9, pp. 392-405, 2008.

[3] S. Han, G. Tang, Y. Chen, and X. Yang, "Optimal vibration control for vehicle active suspension discrete-time systems with actuator time delay," Asian Journal of Control, vol. 15, no. 6, pp. 1579-1588, 2013.

[4] H. Li, J. Yu, C. Hilton, and H. Liu, "Adaptive sliding-mode control for nonlinear active suspension vehicle systems using TS fuzzy approach," IEEE Transactions on Industrial Electronics, vol. 60, no. 8, pp. 3328-3338, 2013.

[5] S.-Y. Han, C.-H. Zhang, and G.-Y. Tang, "Approximation Optimal Vibration for Networked Nonlinear Vehicle Active Suspension with Actuator Time Delay," Asian Journal of Control, vol. 19, no. 3, pp. 983-995, 2017.

[6] A. Chamseddine, H. Noura, and M. Ouladsine, "Design of minimal and tolerant sensor networks for observability of vehicle active suspension," IEEE Transactions on Control Systems Technology, vol. 17, no. 4, pp. 917-925, 2009.

[7] W. Sun, H. Pan, Y. Zhang, and H. Gao, "Multi-objective control for uncertain nonlinear active suspension systems," Mechatronics, vol. 24, no. 4, pp. 318-327, 2014.

[8] P. Brezas and M. C. Smith, "Linear quadratic optimal and risk-sensitive control for vehicle active suspensions," IEEE Transactions on Control Systems Technology, vol. 22, no. 2, pp. 543-556, 2014.

[9] C. Gohrle, A. Schindler, A. Wagner, and O. Sawodny, "Design and vehicle implementation of preview active suspension controllers," IEEE Transactions on Control Systems Technology, vol. 22, no. 3, pp. 1135-1142, 2014.

[10] Z. Ming, N. Hong, and R. Zhu, "Stochastic optimal control of flexible aircraft taxiing at constant or variable velocity," Nonlinear Dynamics, vol. 62, no. 1-2, pp. 485-497, 2010.

[11] J. Lei, Z. Jiang, Y.-L. Li, and W.-X. Li, "Active vibration control for nonlinear vehicle suspension with actuator delay via I/O feedback linearization," International Journal of Control, vol. 87, no. 10, pp. 2081-2096, 2014.

[12] J. Zhu, M. Spiryagin, and L. Wang, "Nonlinear control of vehicle active suspensions," International Journal of Digital Content Technology and its Applications, vol. 6, no. 2, pp. 94-101, 2012.

[13] G. Koch and T. Kloiber, "Driving state adaptive control of an active vehicle suspension system," IEEE Transactions on Control Systems Technology, vol. 22, no. 1, pp. 44-57, 2014.

[14] Z. Xiao and X. Jing, "Frequency-Domain Analysis and Design of Linear Feedback of Nonlinear Systems and Applications in Vehicle Suspensions," IEEE/ASME Transactions on Mechatronics, vol. 21, no. 1, pp. 506-517, 2016.

[15] S. Wen, M. Z. Q. Chen, Z. Zeng, X. Yu, and T. Huang, "Fuzzy Control for Uncertain Vehicle Active Suspension Systems via Dynamic Sliding-Mode Approach," IEEE Transactions on Systems, Man, and Cybernetics: Systems, vol. 47, no. 1, pp. 24-32, 2017.

[16] M. Moradi and A. Fekih, "Adaptive PID-sliding-mode faulttolerant control approach for vehicle suspension systems subject to actuator faults," IEEE Transactions on Vehicular Technology, vol. 63, no. 3, pp. 1041-1054, 2014.

[17] J. Lei, "Optimal vibration control for uncertain nonlinear sampled-data systems with actuator and sensor delays: Application to a vehicle suspension," Journal of Dynamic Systems, Measurement, and Control, vol. 135, no. 2, Article ID 21021, 2013. 
[18] G.-Y. Tang, "Suboptimal control for nonlinear systems: a successive approximation approach," Systems and Control Letters, vol. 54, no. 5, pp. 429-434, 2005.

[19] S.-Y. Han, D. Wang, Y.-H. Chen, G.-Y. Tang, and X.-X. Yang, "Optimal tracking control for discrete-time systems with multiple input delays under sinusoidal disturbances," Journal of Control, Automation, and Systems, vol. 13, no. 2, pp. 292-301, 2015.

[20] M. Bohner, T. S. Hassan, and T. Li, "Fite-Hille-Wintner-type oscillation criteria for second-order half-linear dynamic equations with deviating arguments," Indagationes Mathematicae, 2017.

[21] L. Wang, B. Yang, Y. Chen, X. Q. Zhang, and J. Orchard, "Improving neural-network classifiers using nearest neighbor partitioning," IEEE Transactions on Neural Networks and Learning Systems, 2016.

[22] Z. Yu, Y. Liu, X. Yu, and K. Q. Pu, "Scalable distributed processing of $\mathrm{k}$ nearest neighbor queries over moving objects," IEEE Transactions on Knowledge and Data Engineering, vol. 27, no. 5, pp. 1383-1396, 2015.

[23] H. M. Ngwangwa, P. S. Heyns, F. J. J. Labuschagne, and G. K. Kululanga, "Reconstruction of road defects and road roughness classification using vehicle responses with artificial neural networks simulation," Journal of Terramechanics, vol. 47, no. 2, pp. 97-111, 2010.

[24] J. Lin, "Identification of Road Surface Power Spectrum Density Based on a New Cubic Spline Weight Neural Network," Energy Procedia, vol. 17, pp. 534-539, 2012.

[25] S.-Y. Han, Y.-H. Chen, and G.-Y. Tang, "Fault diagnosis and fault-tolerant tracking control for discrete-time systems with faults and delays in actuator and measurement," Journal of The Franklin Institute, vol. 354, no. 12, pp. 4719-4738, 2017.

[26] S.-Y. Han, Y.-H. Chen, and G.-Y. Tang, "Sensor fault and delay tolerant control for networked control systems subject to external disturbances," Sensors, vol. 17, no. 4, article no. 700, 2017.

[27] H. Du and N. Zhang, "Ho control of active vehicle suspensions with actuator time delay," Journal of Sound and Vibration, vol. 301, no. 1-2, pp. 236-252, 2007. 


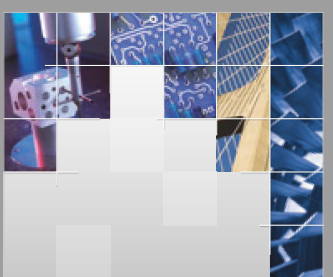

\section{Enfincering}
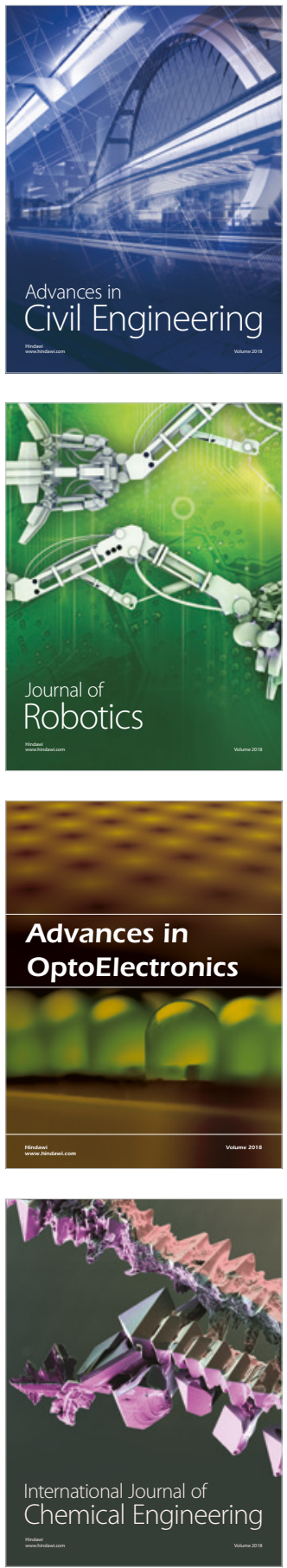

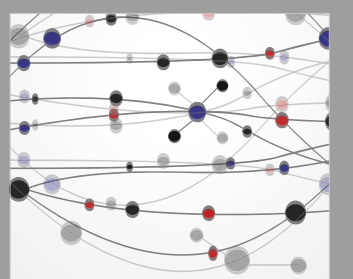

\section{Rotating \\ Machinery}

The Scientific World Journal

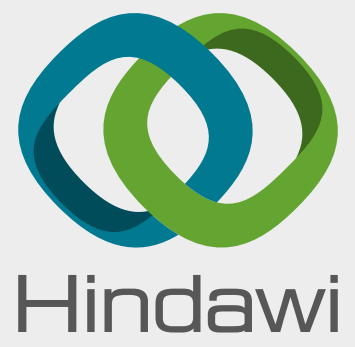

Submit your manuscripts at

www.hindawi.com
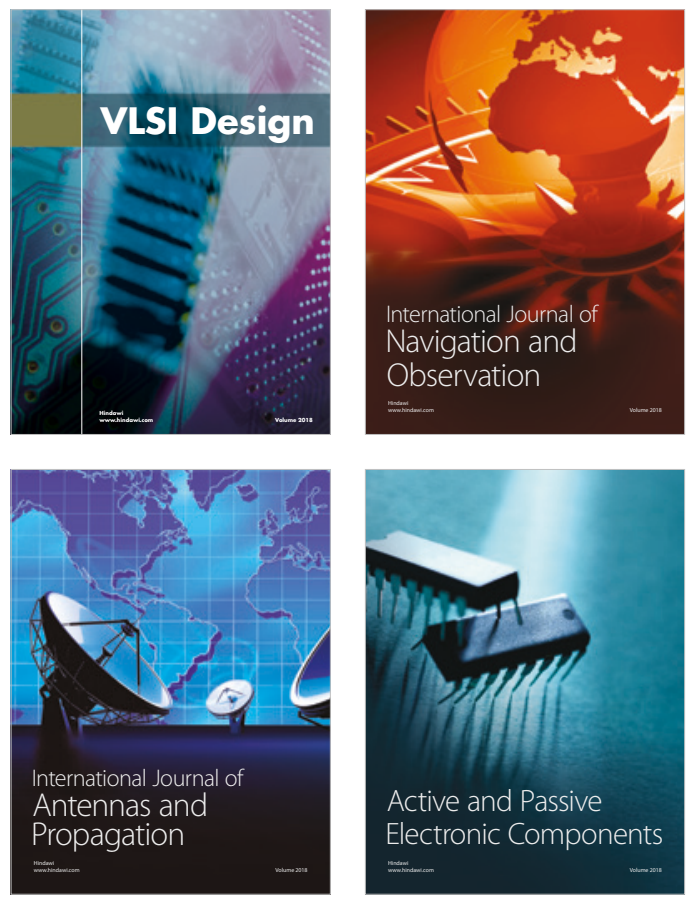
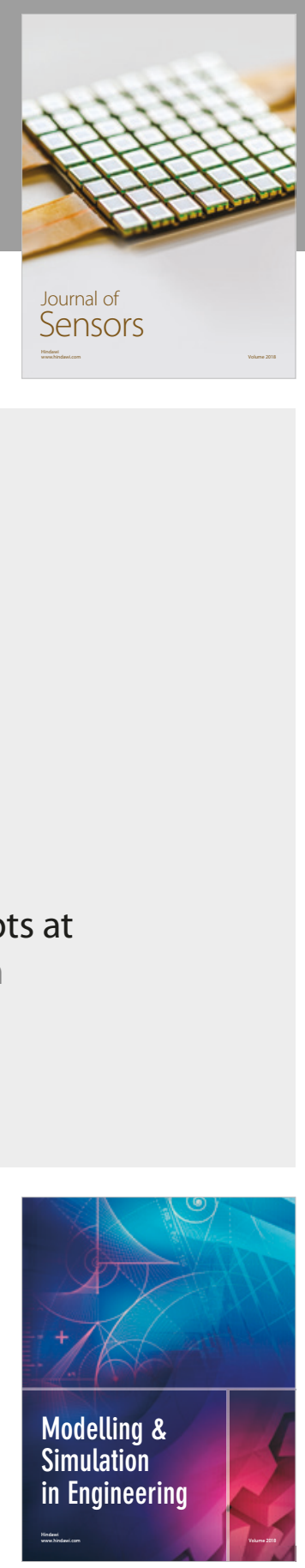

\section{Advances \\ Multimedia}
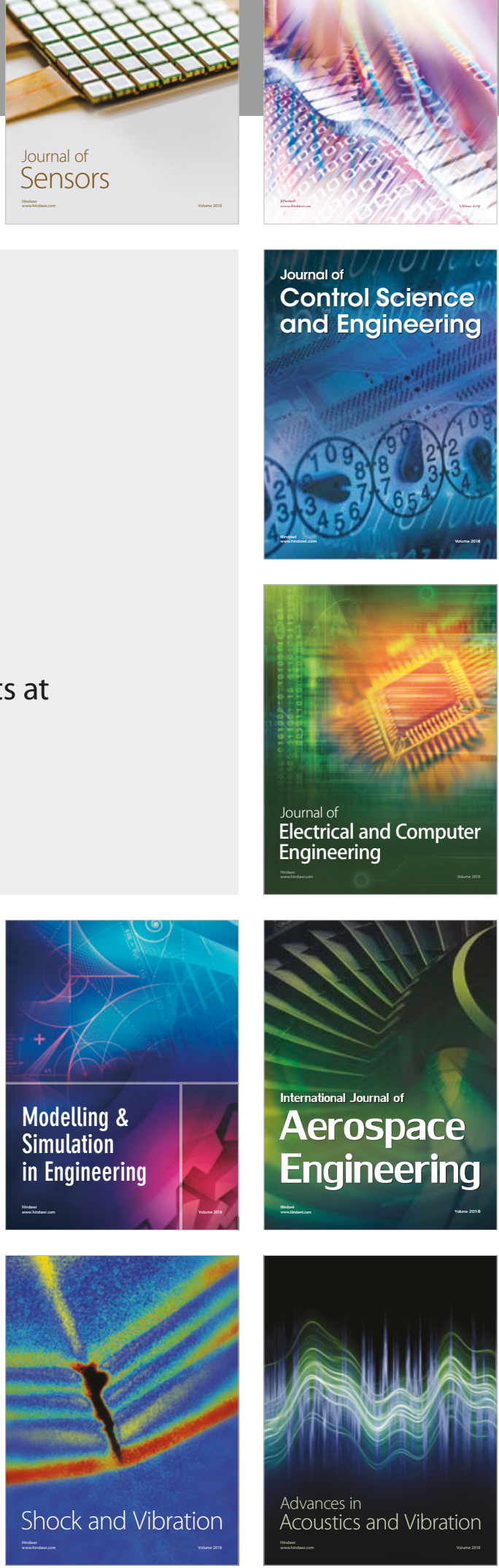